\title{
Personalized Life Cycle Assessment - Reflecting Individuality within the Methodological Framework ${ }^{\text {is }}$
}

\author{
Ann-Kathrin Briem ${ }^{1, *}$, Thomas Betten ${ }^{1}$, and Daniel Wehner ${ }^{2}$ \\ ${ }^{1}$ Institute for Acoustics and Building Physics IABP, University of Stuttgart, Stuttgart, Germany \\ ${ }^{2}$ Fraunhofer Institute for Building Physics IBP, Stuttgart, Germany
}

Received: 20 August 2019 / Accepted: 21 January 2020

\begin{abstract}
Growing environmental awareness in society increasingly influences individual everyday decisions, such as which product to buy or how to sustainably use it. Yet, available information to support these decisions is often limited, or difficult to understand particularly regarding sustainability. Effective ways of communicating environmental impacts of individual decisions are required to close this gap. While Life Cycle Assessment (LCA) is an established tool to evaluate environmental impacts of products and services and support environmental decision-making, the results are typically standardized and based on statistical or averaged data. However, for individuals, this information might be irrelevant, as it neglects personal situation, behavior, information need, or individual level of expertise. In tackling those central issues of personalization in LCA, this article focuses on two main questions: How can individual aspects be addressed in LCA and at which stages of the methodology can LCA be personalized? For this purpose, the ISO 14040/44 standards are analyzed regarding individuality, and current approaches in literature are presented. In an explorative approach, this research identifies two general approaches of personalizing LCA. A personalized Life Cycle Inventory (LCI) enables evaluating the environmental impacts of personal(ized) products and conditions. A broader personalization approach based on the flexibility of the methodological framework of LCA aims at providing understandable and relevant results for individual stakeholders. This article provides an overview, outlines key aspects of this vision, and points out further research needs to bring the concept into application.
\end{abstract}

Keywords: mass personalization / life cycle assessment / individuality / environmental awareness / pro-environmental behavior

\section{Introduction}

Climate change and other environmental impacts are not only in the focus of scientists and politicians but also being discussed in society in general. There is a growing interest in the matter and increasing environmental awareness. More and more individuals start or continue to change their lifestyle with the aim of being more environmentally friendly $[1,2]$. Decisions with impacts on the environment are made by individuals on a daily basis, such as which product to buy or how to use it in a sustainable manner. However, the available information to support these decisions is often limited, not reliable, difficult to understand and thus simply based on gut feeling. Therefore, new and effective ways of communicating the environmental impacts of individual decisions are required.

\footnotetext{
13th International Conference on Society \& Materials, SAM13, Pisa, 20-21 May 2019

* e-mail: ann-kathrin.briem@iabp.uni-stuttgart.de
}

Life Cycle Assessment (LCA) is an established and standardized methodology to assess the environmental impacts of product systems and services over their life cycle $[3,4]$. The information gained from a LCA study can be used to guide decision-makers regarding environmental sustainability. LCA is a flexible tool and widely applicable. On the one hand, this flexibility can lead to inconsistencies between studies, a fact that is addressed in official guidance documents, such as [5]. On the other hand, it also provides the possibility to adapt a study to very specific boundary conditions. Implementing LCA and interpreting the results requires expert knowledge, thus, it is commonly applied and commissioned by relatively large institutions (e.g. corporations or public stakeholders). Furthermore, state of the art LCA studies provide mainly inflexible results tailored to the specific study. For private individuals this information might be too generalized to be truly relevant, hard to understand or difficult to relate to. Considering these individuals as the target audience and adapting the framework of the LCA study, however, could provide the necessary information to foster environmentally preferable behavior. 
Table 1. Overview of keywords found in ISO 14040 and 14044.

\begin{tabular}{lllll}
\hline Framework & $\begin{array}{l}\text { Length of the } \\
\text { document (pages) }\end{array}$ & \multicolumn{2}{c}{$\begin{array}{c}\text { Number of times the word stem is mentioned in the document } \\
\text { "Individual" }\end{array}$} & "Personal" \\
\hline ISO 14040 [3] & 20 & 6 & 0 & 3 \\
ISO 14044 [4] & 46 & 16 & 1 & 3 \\
\hline
\end{tabular}

People are different regarding their information need, expertise, interests, environmental awareness, situation, location, et cetera. These aspects have to be considered in order to successfully personalize LCA. Individually adapted LCA studies could provide easily understandable results, enable informed decision-making and possibly increase environmental awareness, as well as encourage pro-environmental behavior. Depending on the aim of the study, different methods are suitable. Personalizing LCA to support a private person in a daily situation requires a different scope than supporting the traditional users of LCA, such as professional stakeholders. This paper considers and outlines two different personalization approaches for LCA. In the first case of a private individual, adapting the framework of the study would serve the aim of providing relevant and understandable information for this person. Moreover, specific data reflecting this individual's behavior would improve the results regarding their relevance to him or her. In the second case, adapted input data reflecting the individual situation could be sufficient to guide a professional without adjusting the standard framework, i.e. the way the results are presented. Taking individual aspects into account, for example data regarding the user behavior, can support decision-making on a professional level as well, not only the respective individual or target group.

This process of tailored LCAs becomes particularly relevant when looking at current megatrends, such as individualization and mass personalization. Enabled by technological advancements and digitalization, these trends change the way products are manufactured [6]. Consequently, this growing product variability and the influence of the user lead to high uncertainties in a standardized LCA. The effects are particularly relevant for the use phase [7]. Average or general results become less capable for supporting specific individual decisions. New ways to assess the environmental impacts of individual(ized) or personal(ized) products and services through LCA need to be found. Moreover, it will be key to take into account the specific production parameters and individual use patterns.

In order to provide an overview of the central issues of LCA for and of personalized products, this article focuses on two main questions: How can individual aspects be addressed in LCA and at which stages of the methodology can LCA be personalized?

To answer these questions, the ISO 14040/44 standards are analyzed regarding the consideration of individuality in the documents. The state of the art is presented based on literature in Section 3 and in context with the explorative approach of this work. The possibilities of both, personalizing the Life Cycle Inventory (LCI) of a study, as well as personalizing the choices within the methodological framework of LCA are explored and described. The findings are discussed and conclusions drawn regarding further research needs.

\section{Analyzing individuality in ISO 14040 and ISO 14044}

As a first insight into the possibilities for personalization in the methodology of LCA, the ISO 14040 [3] and ISO 14044 [4] standards are analyzed with regards to their referencing of words describing personalization. For this analysis, the stems of the keywords "individual", "personalized" and "customized" are searched for in order to identify these keywords and their derivatives. The aim is to identify those parts of the documents that address individual aspects. The findings are summarized in Table 1 and discussed in the following sections.

The word "individual" and its derivatives can be found 6 times in ISO 14040. Most cases do not refer to individual persons, except for the definition of "interested party" as "individual or group concerned with or affected by the environmental performance of a product system, or by the results of the life cycle assessment". Another section in the standard reflecting personal individuality to a certain extent refers to the fact that "There is no single solution as to how LCA can best be applied within the decision-making context" and further states that "LCA may be used for a broad spectrum of applications. The individual use, adaptation and practice of LCA for all potential applications are based on this International Standard and on ISO 14044".

Words stemming from "personal" are not mentioned in ISO 14040. The three mentioned words containing "custom" in both ISO 14040 and ISO 14044 are mentions of "customers" regarding the definition of service. Therefore, ISO 14040 mentions the broad applicability of LCA but is inconclusive regarding the possibilities to address individuality within the methodological framework.

ISO 14044, being the more application focused guideline document for LCA, contains the word stem "individual" 16 times. Again, most cases do not refer to individual persons. Next to the same definition and explanation mentioned in ISO 14040, however, ISO 14044 mentions "different individuals" in the context of the optional elements of Life Cycle Impact Assessment (LCIA). Ranking, as one option of grouping, and weighting are based on value-choice: "Different individuals, organizations and societies may have different preferences; therefore it is possible that different parties will reach 
different ranking results based on the same indicator results or normalized indicator results." The same is stated for weighting.

"Personal" is mentioned one time in ISO 14044 when defining the reporting requirements if grouping is included in the LCA. Next to clearly stating that grouping is based on value-choice, a justification has to be given regarding "the criteria used for normalization and grouping (these can be personal, organizational or national value-choices)". These examples show that individuality is recognized by the standards, as individual preferences and personal criteria are explicitly mentioned. The standards provide the flexibility to include individual preferences or aspects into the framework by utilizing these optional elements.

\section{Addressing individuality with and within LCA}

Two levels of personalization of LCA were identified. One option is implementing a (standard) LCA of a personalized product or individual situation. Here, individuality is addressed with LCA. The second option is personalizing the chosen goal and scope within the methodological approach focusing on the impact evaluation and result presentation with the aim of providing easily understandable and relevant results. As discussed in Section 2, the methodology of LCA according to ISO 14040/44 is flexible and allows individualizing the implementation of a study while still being compliant with the standards. In this case individuality is addressed within the framework of LCA. Both scopes are discussed in detail in the next sections.

\subsection{Scope: Input data-Personalized product life cycle inventory}

When looking at the four stages of LCA according to ISO 14040/44, the Life Cycle Inventory is when individual data and information have to be available to implement LCA of a personalized product or for an individual situation. To a certain extent the inventory is always unique, as parameters and data sets are chosen to reflect the goal and scope of the study. However, average data is often used for the LCI, either because it serves the goal of the study, to achieve generality or simply because of a lack of specific data. For example, Matuštík and Kočí (2019) [8] investigate personal consumption for average Czech inhabitants. The authors point out that not including behavioral aspects is one limitation of the study, but at the same time ensures wider applicability. The focus is clearly put on finding general patterns of consumption behavior, not individual assessments. In contrast, Arena et al. (2017) [9] present a regional environmental footprint calculator using individual data of daily habits. This research work clearly focuses on pointing out individual user's consumption patterns and related reduction potentials.

The possibilities to individualize the LCI can be located when looking at a typical product life cycle as depicted in Figure 1. The users' influence on the individual stages

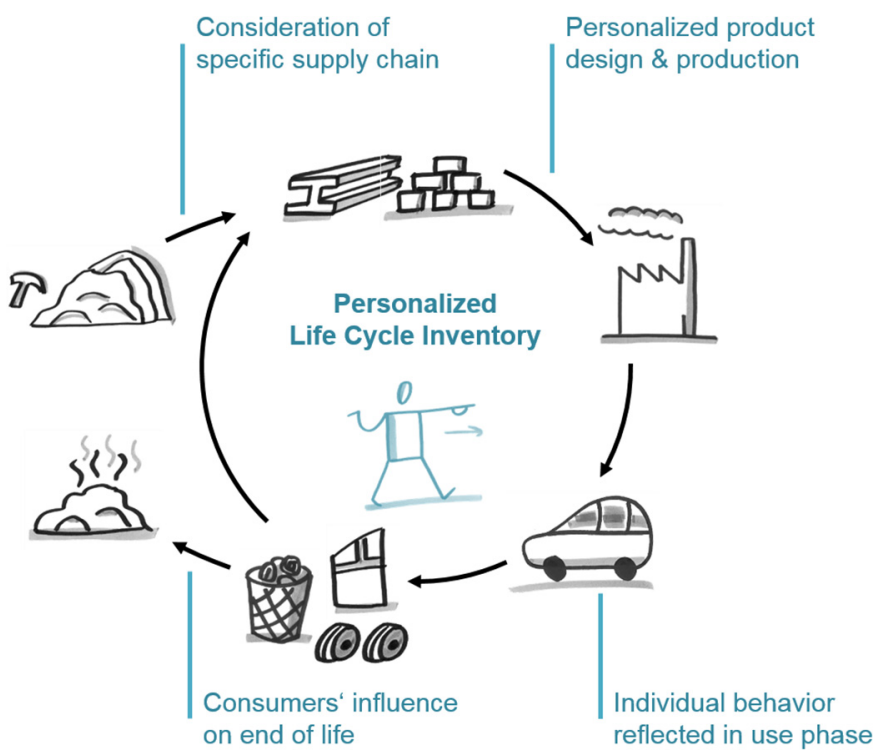

Fig. 1. Possibilities to personalize the Life Cycle Inventory.

(resource extraction, production, use, end of life) and the respective input data is explained in the following paragraphs.

The most obvious influence of the user lies in the use phase, where behavioral patterns strongly affect the environmental performance of a product. In many areas the main life cycle impacts originate from the use phase, such as for standard buildings $[10,11]$ and personal mobility $[12,13]$. The exact share of these impacts strongly depends on the user behavior. Taking individual usage data into account reduces uncertainties and produces relevant results for the specific user as well as for other stakeholders. For example, Briem et al. (2018) [14] investigate the influence of the individual driving behavior on the carbon footprint of different passenger vehicle configurations and reveal that the differences of the overall environmental performance vary widely. A small battery electric vehicle might be the most climate-friendly choice for one person, while a compact diesel vehicle might be the better choice for another person under the individual conditions. Furthermore, considering different potential user behaviors and the respective influence on the environmental performance of a product in the product design phase and tailoring the developments to the target group, can lead to significant benefits. Betten et al. (2019) [7] describe the potential impacts along the use case of a development measure of a lightweight component in the automotive sector. In this example the variability is captured by approx. 3.5 million single scenarios of possible combinations of development measures and user behaviors and visualized in an interactive tool.

This example gives an insight on how advanced methods for data collection, mining and analytics enable the investigation of individual data on a larger scale. With these methods, a single LCA study can provide results for a very large number of scenarios. The level of detail of the available and analyzed data used in studies varies. Most studies rely on made up data, while realistic user data 
would be preferable. However, a lack of specific individual data can be addressed by closing the gap with available data (e.g. statistical, average, etc.).

One method to model user behavior is agent-based modelling. For example, Saner et al. (2013) [15] use agentbased modelling and LCA to assess the environmental impacts of over 3000 individual households in a Swiss community. Based on statistical data, the developed model is used to calculate individual results per household for land-based mobility instead of average values. The heating demand is based on calculations of the hourly heat flux of each building. Walzberg et al. (2019) [16] use an agentbased LCA approach to assess the potential of 100 Canadian smart homes. The authors identify human behavior and pro-environmental behavior change as large influencing factors in this context. Furthermore, they mention as one limitation of the study the use of data from different contexts, such as country-average statistical data instead of specific regional or local data. However, the agent-based LCA approach enables the investigation of complex systems.

Knowing the user and his or her behavior can aid product developers and designers, as mentioned above and shown by Betten at al. (2019) [7]. On top of that, the user might co-create or configure his or her own product following the trend of mass customization. This trend offers potentials but also poses risks for the environmental sustainability of these products $[17,14]$. The individual configuration of the customized product has to be taken into account in the LCI. A modular LCA approach can reduce the arising complexities, as suggested by Fornasiero et al. (2017) [18]. Furthermore, the authors integrate supply chain management into their environmental assessment approach.

Even with customized product design and manufacturing, individual users of a product usually have little to no influence on the supply chain. In a few single cases, however, the user can currently influence the supply chain by choosing a specific (standardized) product. For example, some companies try to establish strictly sustainable supply chains, such as Fairphone and Shiftphone on the phone market $[19,20]$. Hankammer et al. (2016) [21] investigate the smartphone purchasing behavior of students depending on sustainability aspects and customization options. The results show that environmental and social sustainability aspects of products and their supply chain are important influencing factors on the purchasing choice. A path of development could be the possibility for users to customize the supply chain of a product according to their values. Consequently, the specific conditions and actual supply chain could be integrated into a personalized LCI. The challenge at this point is that little background data is currently available in commercially available data bases and LCA practitioners are forced to work with the limited data sets mostly reflecting the global situation.

Finally, the user can strongly influence the lifetime and end of life phase of a product. By taking good care or repairing products, the lifetime can be extended. In contrast, through heavy usage or neglected maintenance, the lifetime could be shortened. Once the product is no longer usable, the user might hold on to it for a while keeping it from re-entering the value chain. If the user properly discards the product, it can enter a path of recycling or disposal; if it is not properly disposed of, the product might not be recycled and ends up as waste. In summary, the influence of the user on the time and path of the end of life stage is high. Therefore, the individual situation can be integrated in the LCI to reflect the situation correctly.

There are many options to individualize the LCI in order to perform LCA of a personalized product or to reflect an individual situation. Some of these options are being used in current literature, but so far without a focus on addressing individuality systematically. Mostly, examples can be found where individual data was available and the study focused on a specific case, rather than having the aim of generating generally applicable results. Depending on the stakeholder, the relevance of implementing individual aspects into the LCI differs. The importance of different life cycle phases varies as well depending on the aim of the study. Furthermore, new technological possibilities, such as big data analytics, enable the implementation of many specific cases at once and easier interpretation of the results.

\subsection{Scope: Goal and scope, evaluation and interpretation}

Personalizing the choices within the methodological framework opens new possibilities. The overall aim of addressing individuality within LCA is to provide understandable and individually relevant results, thus, enabling informed decision-making. This scope of personalization is wider than the one discussed in the previous section, however, both scopes go hand in hand, as a personalized LCI can be part of a personalized LCA, as shown in Figure 2. Moreover, it is most likely essential (but not necessary) to include a personalized LCI into a personalized LCA.

Starting with goal and scope, individual or personal goals can be taken into account when conducting LCA. The system boundary can be chosen accordingly to include individually relevant parts of the product system to be studied or exclude non-relevant parts accordingly. Of course, scientific soundness has to be upheld and cut-off criteria considered. By considering individual aspects from the beginning, relevant results can be produced. The goal and scope is individual by definition. Consequently, one might argue that LCA is in itself personalized. This topic is part of the discussion in Section 4.

The ISO standards mention value-choice based on individual preferences regarding some aspects of the Life Cycle Impact Assessment (LCIA) phase (cf. Sect. 2). One approach to personalize the results is using an individual set of impact categories based on preference, area of interest or environmental awareness of a person (organization, society or other stakeholder). Bai et al. (2018) [22] investigate the understanding of stakeholders regarding different impact categories by combining LCA and conjoint analysis. The results yield the relative importance of each investigated impact category as well as the overall preferences of the interviewed stakeholders. The method can support understanding the reasons behind certain 


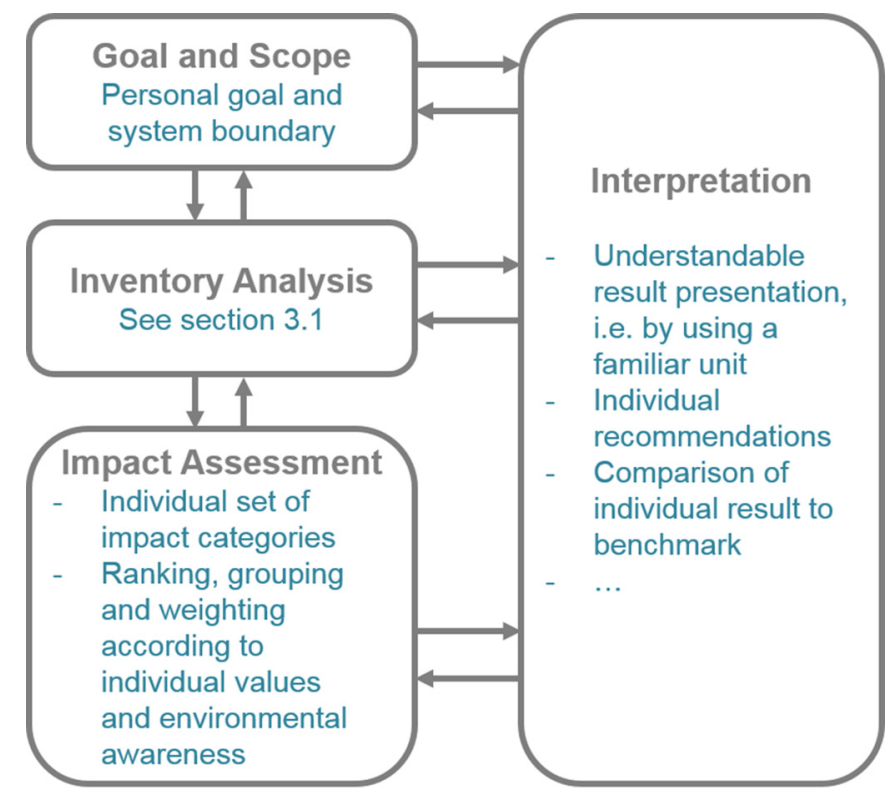

Fig. 2. Possibilities to personalize the methodological framework of LCA.

decisions. Using this method and knowledge, stakeholders could be informed better, for example by providing detailed results for their preferred impact categories. In order to not omit anything, they could be provided with additional information about other impact categories they might not be familiar with.

The optional elements of ranking, grouping and weighting go further, as they are the ones requiring value-choice. Particularly weighting can be a good option to provide understandable results by creating a personalized single-point indicator. However, developing wellsuited weighting factors is challenging. These factors could be based on measurable values, such as the environmental awareness. Itsubo et al. (2018) [23] develop weighting factors based on the environmental awareness in the G20 countries. The weighting is applied to four areas of protection. Next to differences in environmental awareness on country level, varying individual differences can be found within the countries. Some countries show a higher dispersion in personal environmental awareness than others. For an individual weighting of results, for example for different areas of protection as shown by Itsubo et al., the individual values could be used. Using weighting to yield a single-score indicator relevant to the individual could be a useful way to communicate LCA results to non-experts.

In the end, the interpretation phase is key to communicating the obtained results effectively. A familiar unit can be helpful, such as converting $\mathrm{CO}_{2}$-equivalent in "steak-equivalent" or "distance-travelled-by-plane". The latter is used by Bakås et al. (2018) [24] to illustrate the $\mathrm{CO}_{2}$-savings of a configurable recycling station. The savings are converted into a number of flights between Oslo and Trondheim. This approach enables non-LCAexperts to grasp the dimensions of the results better. Depending on the target audience and interested party of the LCA study, this approach might be more or less suitable.
The regional environmental footprint calculator "YUPI ${ }^{\circledR, " ~ b y ~ A r e n a ~ e t ~ a l . ~(2017) ~[9] ~ i s ~ a ~ t o o l ~ t h a t ~ e n a b l e s ~}$ the calculation of the ecological footprint, carbon footprint and water footprint of individuals. The results are also presented using familiar units. The ecological footprint (in hectares) is converted into "planets"; the carbon footprint is presented as the number of trips by car to the United States (from Argentina) and the water footprint is compared to the volume of an Olympic swimming pool. Next to using familiar units, other aspects of individualization are implemented in the calculator as well, such as recommendations on how to lower the personal impacts in daily life. These recommendations are general in the current version of the calculator, but are to be improved and personalized in a newer version, according to the authors.

Generally, the character of recommendations varies depending on the target audience and the questions to be answered by the study as defined in goal and scope. Individualized recommendations based on the results of a LCA study, can provide clearer and more relevant guidance than general recommendations. Another approach of interpreting the results is comparing them to benchmark values. Similar to a familiar unit, the dimension of the results can be better understood by creating a reference point. For familiar units the reference is a relatable measure; for benchmarks the personal results are put in relation to the results of others. By using benchmarks, the results are put into context and no information is lost due to the personalization.

An example for an approach to encourage proenvironmental behavior with personalized recommendations is presented by Anagnostopoulou et al. (2018) [25]. A mobile application for urban route planning suggests different route options based on the user's personality. Persuasive messages are displayed to encourage more environmentally-friendly transport modes. The messages are either based on suggestion, comparison with other users or comparison with the user's past performance (selfmonitoring). The application remembers to which type of message the user responded positively and adjusts future messages accordingly.

\section{Discussion and outlook}

The personalization of LCA is promising, as it unlocks potentials for effective communication and provides relevant results to individuals. Two approaches were presented in this work. The first described scope of personalization focusses on the input data, the second on personalized result communication.

For evaluating the environmental impacts of personal(ized) products, the life cycle inventory can be adjusted accordingly to reflect individual conditions. Moreover, the individual user behavior can be considered. Combining methods, such as agent-based modelling or techniques of big data analytics, with LCA is promising in this regard. The variability due to user behavior can be addressed by analyzing a large number of scenarios. Some approaches can be found in the literature, but further research is needed to fully unlock the potentials in this area. 
New technological advancements and new methods for data collection, mining and analysis exist and enable the implementation of many specific cases at once and easier interpretation of the results. However, acquiring real and usable data remains a challenge for researchers.

Addressing individuality systematically holds high potentials for understanding the environmental impacts associated with individuals. Behavior associated with high environmental impacts can be identified and mitigation options pointed out. This can be done individually, or on a larger scale to support different stakeholders. As presented above, product designers can benefit from considering usephase aspects in the product development process. Furthermore, this approach can support policy-makers in finding new ways to promote pro-environmental behavior. Depending on the stakeholder and the specific application, the importance of including individual aspects into the different life cycle phases varies as well. As discussed, this approach of a personalized LCI is useful for professional stakeholders, but not exclusively. Private individuals can also benefit from this approach, although, understanding the results might pose a challenge.

The second described scope of personalization supports this aim of effective and understandable communication. This personalization scope focusses on the evaluation of the results aiming at providing better understandable and relevant results. By using the flexibility of the standardized methodological framework, individuals' specific questions can be answered. Integrating an individualized LCI into the more widely personalized LCA framework makes sense and is likely essential, yet, not necessary. The main goal of this approach is presenting relevant results in an easily understandable way to individual target audiences. This audience might be anyone from one private individual to a group of professional stakeholders. The idea is to individualize the LCA and the way the results are communicated for the specific case. One might argue that this is what is done in any LCA study, as goal and scope are always unique and the LCA is implemented accordingly. That would mean that any LCA is already personalized. The struggle to generalize findings from LCA studies would surely support this claim. It is difficult to make clear statements or recommendations based on LCA because the results always represent one specific situation, namely the one defined in the goal and scope, and strongly depend on the analyzed boundary conditions. Often a specific group of people or stakeholders is addressed. This could also be the case of a personalized LCA, depending on the level of personalization.

However, this work explored the many ways LCA can be adjusted to truly address individuality. For example, taking the environmental awareness of a person or group into account is one promising approach. As described above, this is possible on the country level, but could also be done on regional, local or individual level. Assessing the environmental awareness can lead to a better understanding of the target audience. Additionally, the environmental knowledge, LCA expertise or preferences, such as specific impact categories, could be assessed and taken into account. By understanding the target audience better, the results can be better presented and relevant recommendations given.
At the moment, this is the task of the LCA practitioner. In the future, advanced analytical methods could assist with this. Further research is needed in this area.

It is shown that personalizing the methodological framework of LCA is possible, due to the flexibility given by the ISO standards. This article aimed at presenting some ideas and exploring the approaches found in the literature. However, the literature analysis is far from exhaustive. Future research should include a thorough analysis of the literature.

Several methods were identified that could assist in implementing the described ideas. The existing synergies of combining different methods with LCA and the underlying potentials should be further investigated. The different methods to measure the environmental awareness have not been in the focus of this work, but have to be addressed in future research. Developing weighting factors based on values for the environmental awareness depend on finding a suitable method. As psychological methods differ strongly from an engineering approach of measuring data in a product system for LCA, the combination of both is challenging.

\section{Conclusion}

This article analyzed the possibilities to address individuality in LCA by presenting existing approaches and exploring new ideas in this context. First, the framework of the ISO 14040/44 standards was analyzed regarding the consideration of individuality. It was found that the standards recognize individuality and explicitly mention personal criteria in the context of value-choice based elements, such as grouping and weighting. Therefore, the standards provide the flexibility to include individual preferences or aspects into the framework by utilizing these optional elements.

Two approaches of personalizing LCA were elaborated and current approaches in the literature presented in context. A personalized LCI enables evaluating the environmental impacts of personal(ized) products and conditions. A broader personalization approach based on the flexibility of the methodological framework of LCA can provide understandable and relevant results for individual stakeholders.

The sensitization of individuals can play a key role in facing the climate crisis and related environmental issues. Society as a whole and each individual can contribute to change the current situation and move towards a more sustainable future. There is a balancing act between encouraging people by promoting pro-environmental behavior and legally limiting environmentally-damaging behavior by proposing regulations. The approaches presented in this work can aid in this regard, as making people understand the impacts of their actions is a first step towards behavior change. LCA can quantify environmental impacts and support decision-making by providing relevant and scientific numbers.

In conclusion, this article provided an overview of the current state of the art of personalized LCA. The key aspects of this vision were discussed and further research 
directions to bring this concept into application were pointed out. By exploring the possibilities to address individuality with LCA, this work only scratched the surface of a broad topic. The authors believe that there is great potential in further developing these ideas.

Acknowledgements. The authors would like to thank the Ministry of Science, Research and Arts and the Ministry of Economic Affairs, Labor and Housing Construction of the German state of Baden-Württemberg for the funding of this work within the HighPerformance Center "Mass Personalization" in Stuttgart.

The High-Performance Center "Mass Personalization", is a cooperation of the four Fraunhofer institutes in Stuttgart, the University of Stuttgart and industry partners. The focus of the project lies on developing and improving key enabling technologies for Mass Personalization and on ensuring sustainable development in this context [26,27].

More information can also be found at www.masspersonalization.de.

\section{References}

1. Bundesministerium für Umwelt, Naturschutz und nukleare Sicherheit (BMU), Umweltbundesamt (UBA), Umweltbewusstsein in Deutschland 2018, Berlin, 2019

2. P.S. Morrison, B. Beer, Consumption and environmental awareness: Demographics of the European experience, in: $\mathrm{H}$. Shibusawa et al. (Eds.), Socioeconomic environmental policies and evaluations in regional science, New Frontiers in Regional Science: Asian Perspectives 24, Springer, Singapore, 2017

3. Deutsches Institut für Normung, DIN EN ISO 14040: Environmental management - Life cycle assessment - Principles and framework, Beuth Verlag, Berlin, Germany, 2006

4. Deutsches Institut für Normung, DIN EN ISO 14044: Environmental management - life cycle assessment - Requirements and guidelines, Beuth Verlag, Berlin, Germany, 2006

5. European Commission - Joint Research Centre-Institute for Environment and Sustainability: International Reference Life Cycle Data System (ILCD), Handbook - General guide for Life Cycle Assessment-Detailed guidance, 1st ed., EUR 24708 EN, Publications Office of the European Union, Luxembourg, 2010

6. C.R. Boër, P. Pedrazzoli, A. Bettoni, M. Sorlini, "Mass customization and sustainability", Springer, London, 2013

7. T. Betten, R. Bouslama, D. Wehner, V. Uusitalo, Unlocking sustainability potentials in product development through extended knowledge and predictions about the product use phase, in: H. Binz et al. (Eds.), Proceedings of the Stuttgarter Symposium für Produktentwicklung SSP 2019, Stuttgart, Germany, 2019, pp. 223-232

8. J. Matuštík, V. Kočí, Environmental impact of personal consumption from life cycle perspective-A Czech Republic case study, Sci. Total Environ. 646, 177-186 (2019)

9. A.P. Arena, R. Piastrellini, G.N. Barón, B.M. Civit, YUPI ${ }^{\circledR}$, A regional footprint calculator, Int. J. Life Cycle Assess. 22, 546-556 (2017)

10. G.A. Blengini, T. Di Carlo, Energy-saving policies and lowenergy residential buildings. An LCA case study to support decision makers in Piedmont (Italy), Int. J. Life Cycle Assess. 15(7), 652-665 (2010)

11. F. Schlegl, J. Gantner, R. Traunspurger, S. Albrecht, P. Leistner, LCA of buildings in Germany: Proposal for a future benchmark based on existing databases, Energy Build. 19, 342-350 (2019)

12. M. Held, R. Graf, D. Wehner, S. Eckert, M. Faltenbacher, S. Weidner, O. Braune, Abschlussbericht: Bewertung der Praxistauglichkeit und Umweltwirkungen von Elektrofahrzeugen, 2016

13. H.C. Kim, T.J. Wallington, Life Cycle Assessment of vehicle lightweighting: A physics based model to estimate use phase fuel consumption of electrified vehicles, Environ. Sci. Technol. 50(20), 11226-11233 (2016)

14. A.-K. Briem, Proceedings of the 8th International Conference on Mass Customization and Personalization-Community of Europe (MCP-CE 2018), Novi Sad, Serbia, 2018

15. D. Saner, H. Heeren, B. Jäggi, R.A. Waraich, S. Hellweg, Housing and mobility demands of individual households and their life cycle assessment, Environ. Sci. Technol. 47, 59885997 (2013)

16. J. Walzberg, T. Dandres, N. Merveille, M. Cheriet, R. Samsona, Assessing behavioural change with agent-based life cycle assessment: Application to smart homes, Renew. Sustain. Energy Rev. 111, 365-376 (2019)

17. G. Pourabdollahian, F. Steiner, O.H. Rasmussen, S. Hankammer, A contribution toward a research agenda: Identifying impact factors of mass customization on environmental sustainability, Int. J. Ind. Eng. Manag. (IJIEM) 5(4), 169-178 (2014)

18. R. Fornasiero, C. Brondi, D. Collatina, Proposing an integrated LCA-SCM model to evaluate the sustainability of customisation strategies, Int. J. Comput. Integr. Manuf. 30(7), 768-781 (2017)

19. F.P. Revellio, Closing loops in the Circular Economy: A make or buy analysis for the smartphone industry, Master's Thesis, Leuphana University of Lüneburg, Lüneburg, Germany, 2017

20. J. Zwiers, S. Behrendt, E. Göll, Fairphone: Fallstudie im Rahmen des Projekts Evolution2Green - Transformationspfade zu einer Green Economy, Adelphi, Borderstep, IZT, Berlin, 2018

21. S. Hankammer, N. Wang, J. Guo, Consumption trends in China and Germany: An empirical cross-cultural study on the role of sustainability and customizability, in: Proceedings of the 7th International Conference on Mass Customization and Personalization in Central Europe, Novi Sad, Serbia, 2016

22. S. Bai, X. Zhu, X. Wang, N. Ren, Identify stakeholders' understandings of life cycle assessment results on wastewater related issues, Sci. Total Environ. 622-623, 869-874 (2018)

23. N. Itsubo, K. Murakami, K. Kuriyama, K. Yoshida, K. Tokimatsu, A. Inaba, Development of weighting factors for G20 countries-Explore the difference in environmental awareness between developed and emerging countries, Int. J. Life Cycle Assess. 23, 2311-2326 (2018)

24. O. Bakås, B. Sjøbakk, L. Skjelstad, M. Thomassen, Integrating sustainability knowledge in choice navigation, in: Proceedings of the 8th International Conference on Mass Customization and Personalization - Community of Europe (MCP-CE 2018), Novi Sad, Serbia, 2018 
25. E. Anagnostopoulou, E. Bothos, B. Magoutas, J. Schrammel, G. Mentzas, Persuasive interventions for sustainable travel choices leveraging users' personality and mobility type, in: J. Ham et al. (Eds.), Persuasive technology, Proceedings of the 13th International Conference PERSUASIVE 2018, Waterloo, ON, Canada, 2018

26. University of Stuttgart, Baden-Württemberg supports new centre of excellence for "Mass Personalization" at the Stuttgart location. Joint initiative by the University of Stuttgart and the Fraunhofer Institute for personalized products, Press release No. 092, 18.07.2017, Available from https://www.uni-stuttgart.de/en/university/news/press-re lease/Neues-Leistungszentrum-Mass-Personalization-amStandort-Stuttgart/ (Accessed: 28/05/2019)

27. M. Held, D. Wehner, R. Hämmerl, M. Dangelmaier, A.-K. Briem, C. Reiff, F. Wulle, Personalization in the automotive and building sector-Research program of the high-performance center "mass personalization" in Stuttgart, in: Proceedings of the 8th International Conference on Mass Customization and PersonalizationCommunity of Europe (MCP-CE 2018), Novi Sad, Serbia, 2018

Cite this article as: Ann-Kathrin Briem, Thomas Betten, Daniel Wehner, Personalized Life Cycle Assessment-Reflecting Individuality within the Methodological Framework, Matériaux \& Techniques 107, 507 (2019) 